\title{
Expression of HERC4 in Lung Cancer and its Correlation with Clinicopathological Parameters
}

\author{
Wen-Li Zeng ${ }^{1,2 \&}$, Yao-Wu Chen ${ }^{1,2 \&}$, Hui Zhou ${ }^{1 *}$, Jue-Yu Zhou ${ }^{1}$, Min Wei ${ }^{1 *}$, Rong Shi $^{1 *}$
}

\begin{abstract}
Background: Growing evidence suggests that the members of the ubiquitin-proteasome system (UPS) are important for tumorigenesis. HERC4, one component, is a recently identified ubiqutin ligase. However, the expression level and function role of HERC4 in lung cancer remain unknown. Our objective was to investigate any correlation between HERC4 and development of lung cancer and its clinical significance. Materials and Methods: To determine HERC4 expression in lung cancer, an immunohistochemistry analysis of a tissue microarray containing samples of 10 lung normal tissues, 15 pulmonary neuroendocrine carcinomas, 45 squamous epithelial cancers and 50 adenocarcinomas was conducted. Receiver operating characteristic (ROC) curve analysis was applied to obtain a cut-off point of $52.5 \%$, above which the expression of HERC4 was regarded as "positive". Results: On the basis of ROC curve analysis, positive expression of HERC4 was detected in 0/10 $(0.0 \%)$ of lung normal tissues, in 4/15 (26.7\%) of pulmonary neuroendocrine carcinomas, in 13/45 (28.9\%) of squamous epithelial cancers and in 19/50 (38.0\%) of adenocarcinomas. It showed that lung tumors expressed more HERC4 protein than adjacent normal tissues $\left(\chi^{2}=4.675, p=0.031\right)$. Furthermore, HERC 4 positive expression had positive correlation with $\mathrm{pT}$ status $\left(\chi^{2}=44.894, p=0.000\right)$, pN status $\left(\chi^{2}=43.628, p=0.000\right)$, histological grade $\left(\chi^{2}=7.083, p=0.029\right)$ and clinical stage $\left(\chi^{2}=72.484, p=0.000\right)$, but not age $\left(\chi^{2}=0.910, p=0.340\right)$. Conclusions: Our analysis suggested that HERC4 is likely to be a diagnostic biomarker for lung cancer.
\end{abstract}

Keywords: HERC4 - lung cancer - tissue microarray - clinicopathological parameters - diagnostic biomarker

Asian Pac J Cancer Prev, 16 (2), 513-517

\section{Introduction}

Lung cancer is the leading cause of death of cancer world-wide, causing more than 1 million deaths per year (Jemal et al., 2010). It is a disease characterized by uncontrolled cell growth in tissues of the lung. The global cancer burden in annual cases is projected to double by 2050 , and lung cancer is expected to remain the leading cause of all cancer deaths during that time (Brothers et al., 2013). Cigarette smoking remains the main risk factor for lung cancer,

with $85 \%-90 \%$ percent of lung cancer cases caused by active or passive smoking. Conventional treatments for lung cancer are surgery, radiotherapy and chemotherapy. And the choice of method depends on the type, progress and relevant genes of lung cancer. Besides, targeted therapy, as a more accurate and specific treatment, has come into our field of vision for us to choose from. It won't cause any harm to adjacent normal lung cells when it uses specific drugs to identify and target cancer cells.

Recently, genetic, transcriptomic and epigenomic biomarkers are emerging as tools for the early diagnosis of lung cancer both in the screening setting and diagnostic. Furthermore, epigenetics refers to heritable modifications of DNA and associated chromatin components that influence gene expression without altering DNA coding sequence. Two key levels of aberrant epigenetic control are DNA methylation and histone acetylation (Vendetti and Rudin, 2013). A recent study on methylation profiles of a slightly larger set of 15 genes has selected the six most sensitive and specific genes for predicting lung cancer risk (APC, CDH1, MGMT, DCC, RASSF1A and AIM1). And it clearly shows evidence that a more global methylome approach could lead to a more sensitive (75\%) and specific (73\%) biomarker of lung cancer risk from serum DNA (Begum et al., 2011). Considering the high false positive rate of computed tomography of the chest, there is an urgent need to develop biomarkers that can distinguish benign from malignant lesions efficiently.

To some extent, the high mortality in patients with lung cancer ( $80 \%$ to $85 \%$ in five years) results from the lack of effective tools to diagnose the disease at its early stage. However, the sequencing of the human genome together with the technological advances have opened a new era of molecular biomarker development that promises to solve this problem. The ubiquitin-proteasome system (UPS) is the primary pathway responsible for the recognition and degradation of misfolded, damaged, and

${ }^{1}$ Institute of Genetic Engineering, ${ }^{2}$ The First Clinical Medical College, Southern Medical University, Guangzhou, China ${ }^{\star}$ Equal contributors*For correspondence: shirongphd@126.com or weimin78@126.com 
tightly regulated proteins. It also plays crucial roles in DNA repair, cell cycle regulation, cell migration, and the immune response (Melvin et al., 2013). Ubiquitin (Ub) is a small protein composed of 76 amino acids that is highly conserved in eukaryotes (Tomohiro and Mizuno, 1995). Post-translational protein modification by ubiquitin targets the protein to the $26 \mathrm{~S}$ proteasome. Protein ubiquitination requires a cascade of three increasingly diverse enzymes: ubiquitin activating enzyme (E1 enzyme), ubiquitin conjugating enzyme (E2 enzyme) and ubiquitin ligase (E3 ligase) (Deshaies and Joazeiro, 2009). Moreover, The E1-E3 enzymatic cascade is paralleled by several ubiquitin-like proteins (UbL), which has become increasingly apparent that these UbLs play an integral role in the regulation of cellular processes, including transcriptional regulation and DNA repair (Hickey et al., 2012). The unique and vital functions carried out by the UPS in regulating protein levels have made it a very attractive target for novel therapeutics. For instance, dysregulation of protein production in multiple myeloma, particularly antibodies, can create an increased reliance on the proteasome to degrade misfolded or overproduced proteins. Incomplete inhibition of the proteasome proves too stressful for cancer cells, while proving less cytotoxic to healthy cells. This allows a more targeted treatment method and has led to the development of the next generation proteasome inhibitor Carfilzomib (Kuhn et al., 2007). In addition, there are conflicting reports about decreased proteasome activity suspected to play a key role in conformational diseases such as Parkinson's and Huntington's disease(Lindsten and Dantuma, 2003). Researchers are telling us in various aspects that the links between members of the UPS and carcinogenesis ( $\mathrm{Ji}$ et al., 1999; Deng and Scott, 2000). Consequently, the role of the UPS in its regulation has become an area of intense interest.

HERC4 was a recently identified member of the human HERC family of proteins, which consist of six members and all contain one or more RCC1 (regulator of chromosome condensation 1)-like domains (RLD) upstream of a HECT domain. HERC protein serves a dual function. On the one hand, RLD could function as guanine nucleotide exchange factor (GEF) for Ran like $\mathrm{RCC} 1$, which participates in nucleocytoplasmic transport and mitotic spindle formation. On the other hand, the HECT domain was firstly defined in E6-AP-mediated ubiquitination of p53 and performs as ubiquitin ligase (Ji et al., 1999). HERC4 was located in chromosome 10q22, composing of 26 exons. Recent study showed that the selection of tissue and cell types was based on the expression profile for HERC4 and alternative splicing of exons 24 and 25 in HERC4 may result in altered binding to the ubiquitin conjugase, a further possibility for interfering with ubiquitin ligase activity. Additionally, HERC4 was expressed as multiple spliced products with higher mRNA levels in brain and testis (Hochrainer et al., 2005). Moreover, the disruption of the murine HERC4 gene induced a reduction in male fertility related to a defect in the late stages of spermatogenesis, which proved the necessity for proper spermatogenesis and male fertility in mice (Rodriguez and Stewart, 2007).
However, the functional role of HERC4 in other diseases, such as cancer, is still unclear. To address this issue, we decided to investigate whether HERC4 was correlated to the development of lung cancer and clinical significance.

\section{Materials and Methods}

\section{Tissue samples}

We collected 120 paraffin-embedded tissue samples in total from patients with kinds of lung cancers before receiving any treatments in the Nanfang Hospital of Southern Medical University between 2005 and 2012. The paraffin-embedded tissue cases included 10 lung normal tissues, 15 pulmonary neuroendocrine carcinoma, 45 squamous epithelial cancer and 50 adenocarcinoma. And the mean age of the 110 patients (between 14 and 76 years) with kinds of lung cancers was 56.3 years apart from the 10 normal ones and Table 3 showed all details mention above. The Ethic Committee of the Nanfang Hospital had written informed consent of all patients for a protocol to approve us to perform this research.

Construction of tissue microarrays and immunohistochemistry analysis

The standard method for the construction of tissue microarray(TMA) is described as below. Firstly, the representative areas of collected tissues were chosen and punched into duplicate $0.6 \mathrm{~mm}$ diameter cylinders, which would be re-embeded into new paraffin blocks (recipient paraffin block) using a tissue-arraying instrument (Minicore, Mitogen, U.K.) in the right place determined beforehand. Then, for the need of immuohistochemistry analysis, TMA block would be cut into several 5- $\mu \mathrm{m}$ sections. Immunohistochemical (IHC) staining was performed based on standard procedures. For the deparaffinization and rehydration of formalin-fixed and paraffin-embedded tissue array, xylene and different levels of ethanol were applied sequentially. Then, $0.3 \%$ $\mathrm{H}_{2} \mathrm{O}_{2}$ was taken to inhibit the activity of endogenous hydrogen peroxidase. Afterwards, antigen retrieval was carried out with sodium citrate buffer $(\mathrm{pH} \mathrm{6.0)}$ at $100^{\circ} \mathrm{C}$ for 20 minutes. In addition, after blocking with $5 \%$ normal goat serum, tissue array would be incubated with HERC4 primary antibody (Rabbit polyclonal, 1:200, PL laboratories, U.S.A.) at $4^{\circ} \mathrm{C}$ overnight. When finished, it was washed for 3 times with $1 \mathrm{X}$ phosphate buffered saline (PBS) of 10 minutes each time, and then incubated with polymer peroxidase-labeled secondary antibody (Zhongshan biotech, China) for 30 minutes at room temperature. Finally, signal in the tissue array was visualized by using DAB Horseradish Peroxidase Color Development Kit (Beyotime, China) after hematoxylin counterstaining, while phosphate-buffered saline was set as negative control.

\section{Evaluation of immunohistochemistry results and Selection of Cut-off scores}

With a semi-quantitative scoring method, based on the evaluation of the percentage of positive HERC4 staining tumor cells over the total tumor cells, three researchers gave scores independently according to the five percent 
Expression of HERC4 in Lung Cancer and its Correlation with Clinicopathological Parameters

increasing rate method $(0 \%, 5 \%, 10 \%, 15 \%, 20 \% \ldots 100 \%)$ to analyze the immunohistochemical expression of HERC4. Receiver operating characteristic (ROC) curve analysis was taken to get an optimal cut-off score to analyze and distinguish the tumor with HERC4 expression using 0,1-criterion. For the need of ROC curve analysis, we divided the clinicopathological parameters into two parts as follows: pT status (T1-T2, T3-T4), pN status (N0, N1-N2), clinical stage (I-II, III-IV) and histological grade (G0-G1, G2-G3). Each point on the curve was created by plotting the paired sensitivity and specificity of each HERC4 score. The distance of each point to the point $(0.0$, $1.0)$ on the curves were measured and the minimum one were chosen to be the cut-off score. Then, the cut-off score was set as the threshold. When HERC4 score below the threshold, the tumor was defined as "negative". Otherwise, it would be defined as "positive".

\section{Statistical analysis}

All data was analyzed by the SPSS 13.0 software (SPSS, Chicago, IL, USA). Chi-square test was used to estimate the relationship between HERC4 expression and the clinicopathological parameters of the patients with lung cancer, which would be considered statistical significant when the P-value was $<0.05$ (two-tailed test).

\section{Results}

Expression status of HERC4 in tissue samples of lung cancer

IHC was applied to assess the expression status of HERC4 in lung tumors and adjacent lung normal tissues. We mainly observed the immunoreactivity in cytoplasm of cells. Four representative samples with an increasing expression level of HERC4 of both lung tumors and lung normal tissues were showed in Figure 1. Receiver operating characteristic (ROC) curve analysis was taken to analyze the relationships of HERC4 expression status with histological grade, clinical stage, pT status and pN status. Four ROC curves were generated and showed in Figure 2. Based on the analysis results, the point had the shortest distance to the point $(0.0,1.0)$ on the curve of clinical stage, which was determined as the optimal cut-off score. The cut-off score was $52.5 \%$ and above it was defined as positive. According to it, our analysis results showed that $0 / 10(0 \%)$ of lung normal tissues, $4 / 15$ $(26.7 \%)$ of pulmonary neuroendocrine carcinoma, 13/45 $(28.9 \%)$ of squamous epithelial cancer and 19/50 (38\%) of adenocarcinoma were HERC4 positive expression. And details were showed in Table 1. Furthermore, we divided the total 120 tissue samples into two parts. One part included 10 lung normal tissues and the other part contained 110 lung tumor tissues. After analyzing, we found that HERC4 expression increased significantly
A

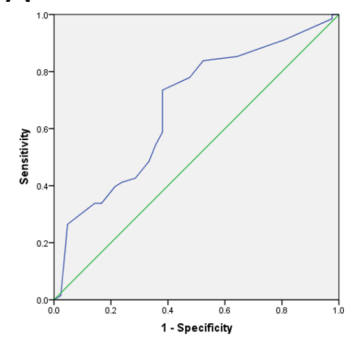

C

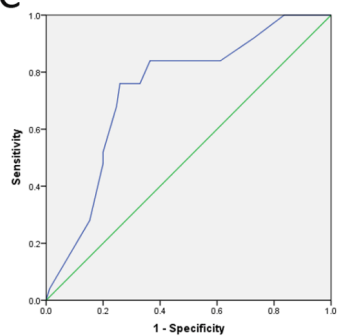

B

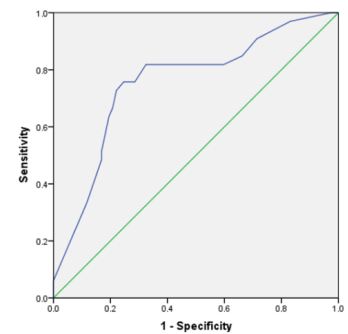

$\mathrm{D}$

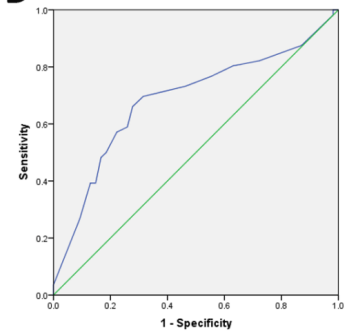

Figure 2. ROC Curves Analysis was Used for the Selection of the Cut-Off Score. Four ROC curves were generated by the sensitivity and specificity of each score: $\mathbf{A})$ histological grade, B) clinical stage, C) pT status, D) pN status
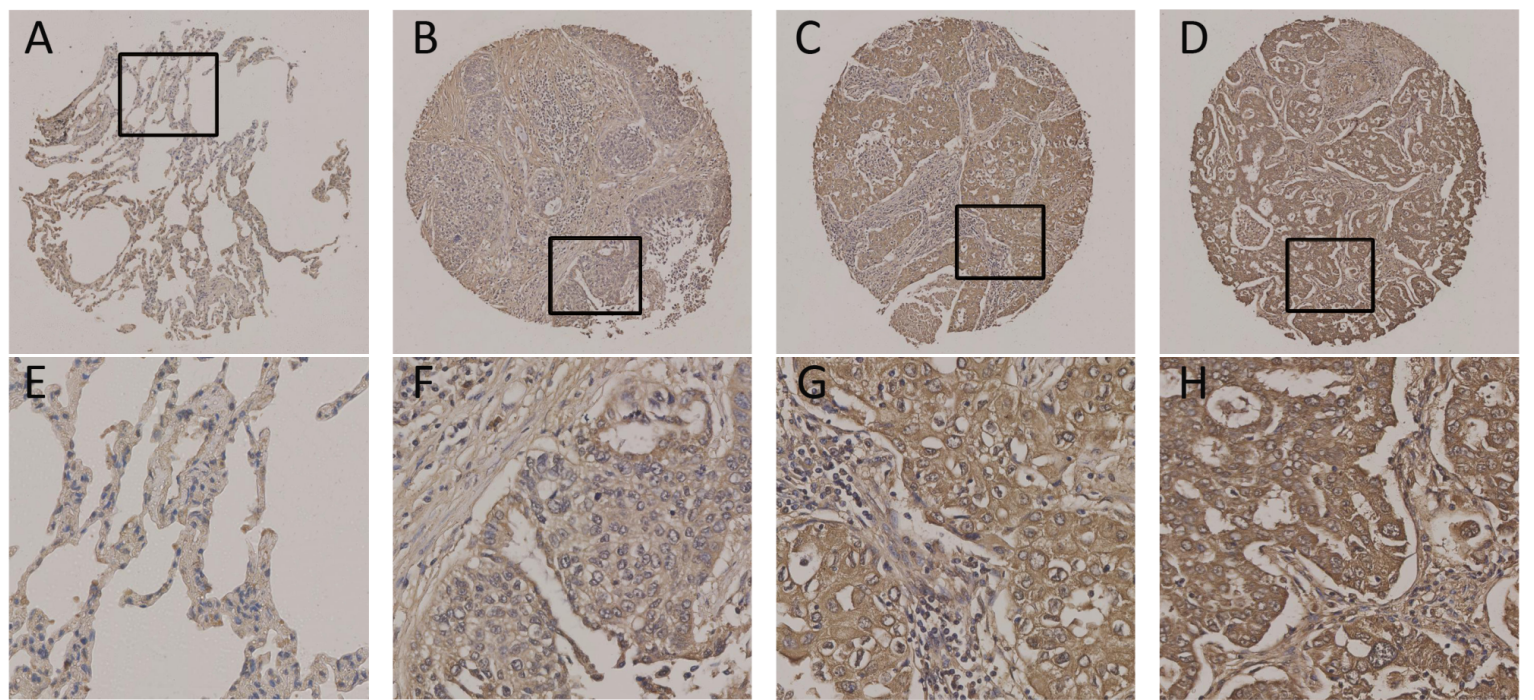

Figure 1. IHC Results of the Expression of HERC4 in Lung Normal Tissues and Lung Tumor Tissues. A) Negative expression of HERC4 was detected in lung normal tissue (case116) (100x). B) 30\% positive expression of HERC4 was detected in a squamous cell carcinoma (case 17) (100x). C) 60\% positive expression of HERC4 was showed in a squamous cell carcinoma (case 31) (100x). D) $90 \%$ positive expression of HERC4 was showed in adenocarcinoma (case 56) (100x). E), F), G) and H) demonstrate the higher magnification (400x) from the area of black box in (A), (B), (C) and (D), respectively 
Table 1. The Expression of HERC4 in Lung Tissues

\begin{tabular}{|c|c|c|c|}
\hline \multicolumn{4}{|c|}{ HER4 staining } \\
\hline & $\begin{array}{c}\text { All } \\
\text { cases }\end{array}$ & $\begin{array}{c}\text { Negative } \\
\text { expression } \\
(\%)\end{array}$ & $\begin{array}{c}\text { Positive } \\
\text { expression } \\
(\%)\end{array}$ \\
\hline \multicolumn{4}{|c|}{ Lung normal tissues } \\
\hline & 10 & $10(100.0)$ & $0 \quad(0.0)$ \\
\hline \multicolumn{4}{|c|}{ Pulmonary neuroendocrine carcinoma } \\
\hline & 15 & $11(73.3)$ & $4(26.7)$ \\
\hline \multicolumn{4}{|c|}{ Squamous epithelial cancer } \\
\hline & 45 & $32(71.1)$ & $13(28.9)$ \\
\hline \multicolumn{3}{|l|}{ Adenocarcinoma } & $19(38.0)$ \\
\hline
\end{tabular}

Table 2. Relationship of HERC4 Expression between Lung Normal Tissues and Lung Tumor Tissues

\begin{tabular}{lccrrr}
\hline \multicolumn{5}{c}{ HERC4 staining } \\
\hline & $\begin{array}{c}\text { Negative } \\
(\%)\end{array}$ & \multicolumn{1}{c}{$\begin{array}{c}\text { Positive } \\
(\%)\end{array}$} & Total & $P$ value \\
\hline Lung normal tissues & $10(100.0)$ & 0 & $(0.0)$ & 10 & 0.031 \\
Lung tumor tissues & 74 & $(67.3)$ & $36(32.7)$ & 110 & \\
\hline${ }^{*} P$ value are from Chi-square test & & & &
\end{tabular}

Table 3. Relationship between HERC4 Expression and Clinicopathological Features in Lung Cancers

\begin{tabular}{|c|c|c|c|c|}
\hline \multirow[t]{2}{*}{ Variables } & \multicolumn{4}{|c|}{ HERC4 staining } \\
\hline & $\begin{array}{c}\text { Negative } \\
(\%)\end{array}$ & $\begin{array}{c}\text { Positive } \\
(\%)\end{array}$ & Total & $P$ value \\
\hline \multicolumn{5}{|l|}{ Age(years) } \\
\hline$\leq 56.3^{\dagger}$ & $38(71.7)$ & $15(28.3)$ & 53 & 0.340 \\
\hline$>56.3$ & $36(63.2)$ & 21 & 57 & \\
\hline \multicolumn{5}{|l|}{ pT status } \\
\hline $\mathrm{T} 1-\mathrm{T} 2$ & $71(83.5)$ & $14(16.5)$ & 85 & 0.000 \\
\hline T3-T4 & $3(12.0)$ & $22(88.0)$ & 25 & \\
\hline \multicolumn{5}{|l|}{$\mathrm{pN}$ status } \\
\hline No & $50(92.6)$ & $4 \quad(7.4)$ & 54 & 0.000 \\
\hline N1 & $24(54.5)$ & $20 \quad(45.5)$ & 44 & \\
\hline $\mathrm{N} 2$ & $0(0.0)$ & $12(100.0)$ & 12 & \\
\hline \multicolumn{5}{|c|}{ Histological grade } \\
\hline G0-G1 & $32(76.2)$ & $10 \quad(23.8)$ & 42 & 0.029 \\
\hline G2 & $34(69.4)$ & $15(30.6)$ & 49 & \\
\hline G3 & $8(42.1)$ & $11 \quad(57.9)$ & 19 & \\
\hline \multicolumn{5}{|c|}{ Clinical Stage } \\
\hline I-II & $71(92.2)$ & $6 \quad(7.8)$ & 77 & 0.000 \\
\hline III-IV & $3(9.1)$ & $30 \quad(90.9)$ & 33 & \\
\hline
\end{tabular}

in the 110 lung tumor tissues compared to the 10 lung normal tissues $\left(\chi^{2}=4.675, p=0.031\right)$. Results were showed in Table 2.

\section{Relation between HERC4 and clinicopathological parameters}

The analysis results of the relationship of HERC4 expression status with different clinicopathological parameters like age, histological grade, clinical stage, pT status, $\mathrm{pN}$ status were showed in Table 3. According to the results, the expression of HERC4 has no significant correlation with age $\left(\chi^{2}=0.910, p=0.340\right)$. But HERC4 expression increased in lung tumor tissues as the histological grade of tumor progressed $\left(\chi^{2}=7.083\right.$, $p=0.029)$, lymph node metastasized $\left(\chi^{2}=43.628, p=0.000\right.$ ), clinical stage developed $\left(\chi^{2}=72.484, p=0.000\right)$ and tumor size largened $\left(\chi^{2}=44.894, p=0.000\right)$.

\section{Discussion}

Rapid development of technological advances have provided various methods for the further study of lung cancer, but its etiology still remain to be clearly defined. Although several sensitive and specific genes for predicting lung cancer risk had been found (Begum et al., 2011), biomarkers with the ability to distinguish benign from malignant lesions were still unable to meet our demand. Last several years, increasing evidence had showed the important role of ubiquitin-proteasome system in tumorigenesis (Ji et al., 1999; Deng and Scott, 2000), which drew our strong attention. HERC4 was recently identified to be a member of HERC family and it had some association with ubiquitin ligase. Studies had confirmed that murine HERC4 lead to a reduction of male fertility, for it could degrade spermatogenesis related proteins by ubiquitin-proteasome pathway (Rodriguez and Stewart, 2007). However, the role of HERC4 in lung cancer and its utility for clinicians needed continuous study. In our study, we paid attention to the differences of HERC4 expression in both lung cancers and adjacent lung normal tissues and we managed to find out the association of HERC4 expression with clinicopathological parameters.

Then, in order to find the exact difference of HERC4 expression in kinds of lung cancers, we performed a tissue microarray containing 120 lung tissue samples of 10 lung normal tissues, 15 pulmonary neuroendocrine carcinoma, 45 squamous epithelial cancer and 50 adenocarcinoma. Then, a scoring system method based on the percentage of positive staining tumor cells over the total tumor cells was taken by us to assess the immunoreactivity of HERC4. In order to obtain a convincing cut-point for IHC evaluation of HERC4, ROC curve analysis was chosen to select optimal cut-off for HERC4 positivity. Afterwards, the paired sensitivity and specificity of each HERC4 score were used to plot the curve. According to the clinicopathological parameters we got from the tissue samples, including histological grade, clinical stage, $\mathrm{pT}$ status and $\mathrm{pN}$ status, different ROC curves were created. Finally, the optimal cut-off point for HERC4 score of each curve was selected and those above $52.5 \%$ was determined as HERC4 positive expression. In this premise, $0 / 10$ $(0.0 \%)$ of lung normal tissues, $4 / 15$ (26.7\%) of pulmonary neuroendocrine carcinoma, $13 / 45$ (28.9\%) of squamous epithelial cancer and 19/50 (38.0\%) of adenocarcinoma showed HERC4 positive expression. Besides, comparing the 10 lung normal tissues to the 110 lung tumor tissues, we detected HERC4 positive expression in $0 / 10(0.0 \%)$ of lung normal tissues and 36/110(32.7\%) of lung tumor tissues $\left(\chi^{2}=4.675, p=0.031\right)$, which suggested upregulation of HERC4 in lung tumor tissues. Moreover, further analysis results showed significant positive correlation between lung cancer and clinicopathological parameters we focused on, such as histological grade $\left(\chi^{2}=7.083, p=0.029\right)$, clinical stage $\left(\chi^{2}=72.484, p=0.000\right)$, pT status $\left(\chi^{2}=44.894, p=0.000\right)$ and $\mathrm{pN}$ status $\left(\chi^{2}=43.628\right.$, $p=0.000)$. 
In conclusion, our analysis results showed that the expression of HERC4 protein in lung tumor tissues were more than that in the adjacent lung normal tissues. And positive expression of HERC4 has obvious positive correlation with histological grade $\left(\chi^{2}=7.083, p=0.029\right)$, clinical stage $\left(\chi^{2}=72.484, p=0.000\right)$, pT status $\left(\chi^{2}=44.894\right.$, $p=0.000)$ and $\mathrm{pN}$ status $\left(\chi^{2}=43.628, p=0.000\right)$, which suggests that HERC4 is likely to be a diagnostic biomarker for lung cancer. As for the mechanisms of how HERC4 functions needs further research.

\section{Acknowledgements}

This work was supported by the National Natural Science Foundation of China (Grant No. 81372154, 81101536, 30901721), and the Scientific Research Foundation for the Returned Overseas Chinese Scholars, Chinese Ministry of Education (the $40^{\text {th }}$ batch), Science and Technology Planning Project of Guangdong Province, China (2013b021800146)

\section{References}

Begum S, Brait M, Dasgupta S, et al (2011). An epigenetic marker panel for detection of lung cancer using cell-free serum DNA. Clin Cancer Res, 17, 4494-503.

Brothers JF, Hijazi K, Mascaux C, et al (2013). Bridging the clinical gaps: genetic, epigenetic and transcriptomic biomarkers for the early detection of lung cancer in the post-national lung screening trial era. BMC Med, 11, 168.

Deng CX, Scott F (2000). Role of the tumor suppressor gene Brcal in genetic stability and mammary gland tumor formation. Oncogene, 19, 1059-64.

Deshaies RJ, Joazeiro CA (2009). RING domain E3 ubiquitin ligases. Annu Rev Biochem, 78, 399-434.

Hickey CM, Wilson NR, Hochstrasser M (2012). Function and regulation of SUMO proteases. Nat Rev Mol Cell Biol, 13, 755-66.

Hochrainer K, Mayer H, Baranyi U, et al (2005). The human HERC family of ubiquitin ligases: novel members, genomic organization, expression profiling, and evolutionary aspects. Genomics, 85, 153-64.

Jemal A, Center MM, DeSantis C, et al (2010). Global patterns of cancer incidence and mortality rates and trends. Cancer Epidemiol Biomarkers Prev, 19, 1893-907.

Ji Y, Walkowicz MJ, Buiting K, et al (1999). The ancestral gene for transcribed, low-copy repeats in the prader-willi/ angelman region encodes a large protein implicated in protein trafficking, which is deficient in mice with neuromuscular and spermiogenic abnormalities. Hum Mol Genet, 8, 533-42.

Kuhn DJ, Chen Q, Voorhees PM, et al (2007). Potent activity of carfilzomib, a novel, irreversible inhibitor of the ubiquitinproteasome pathway, against preclinical models of multiple myeloma. Blood, 110, 3281-90.

Lindsten K, Dantuma NP (2003). Monitoring the ubiquitin/ proteasome system in conformational diseases. Ageing Res Rev, 2, 433-49.

Melvin AT, Woss GS, Park JH, et al (2013). Measuring activity in the ubiquitin-proteasome system: from large scale discoveries to single cells analysis. Cell Biochem Biophys, 67, 75-89.

Rodriguez CI, Stewart CL (2007). Disruption of the ubiquitin ligase HERC4 causes defects in spermatozoon maturation and impaired fertility. Dev Biol, 312, 501-8.

Tomohiro M, Mizuno A (1995). Alteration of lens sulfhydryl groups induced by oxidative stress: Raman spectroscopic study of hydrogen peroxide-treated rat lens. Jpn J Ophthalmol, 39, 130-6.

Vendetti FP, Rudin CM (2013). Epigenetic therapy in non-smallcell lung cancer: targeting DNA methyltransferases and histone deacetylases. Expert Opin Biol Ther, 13, 1273-85. 